\title{
CDR-MAC: A Protocol for Full Exploitation of Directional Antennas in Ad Hoc Wireless Networks
}

\author{
Thanasis Korakis, Member, IEEE, Gentian Jakllari, Student Member, IEEE, and \\ Leandros Tassiulas, Fellow, IEEE
}

\begin{abstract}
In this paper, we propose a new Medium Access Control (MAC) protocol for full exploitation of directional antennas in wireless networks. The protocol introduces a circular directional transmission of the Request To Send (RTS) control packet, spreading around a station information about the intended communication. The stations that receive the directional RTS, using a simple scheme of tracking the neighbors' directions, defer their transmission toward the beams that could harm the ongoing communication. In this way, the proposed protocol takes advantage of the benefits of directional transmissions as the increase of spatial reuse and of coverage range. Additionally, it reduces the hidden-terminal problem, as well as the deafness problem, two main factors for the decrease of the efficiency of directional transmissions in ad hoc networks. The performance evaluation of the protocol shows that it offers a significant improvement in static, as well as mobile, scenarios, as compared to the performance of the proposed protocols that use omnidirectional or directional transmissions.
\end{abstract}

Index Terms-Wireless communications, MAC, directional antennas, IEEE 802.11.

\section{INTRODUCTION}

$A$ D hoc wireless networks have attracted a growing A interest in many application domains, which include commercial applications, military applications, and applications in environments where the presence of an infrastructure network is impossible or not affordable. Currently, the technology of transmitting data over the air is based on omnidirectional antennas; therefore, most of the research in designing Medium Access Control (MAC) protocols has assumed their deployment. The result is a number of protocols, including the industry standard IEEE 802.11 [11], [17], which seems to effectively solve the challenges projected in this environment.

The electromagnetic energy of the signal in omnimode transmission is spread over a large region of space, whereas only a small portion of it is received by the intended receiver. Directional antennas solve this efficiency problem by using $M$ elements. These kinds of antennas transmit in directional mode, which means that electromagnetic waves are enhanced in certain directions and are attenuated in others, resulting in an amplified signal that is directed to

- T. Korakis is with the Electrical and Computer Engineering Department, Polytechnic University, 5 Metrotech Center, Brooklyn, NY 11201.

E-mail:korakis@poly.edu.

- G. Jakllari is with the Department of Computer Science and Engineering, University of California, Riverside, Engineering Building II, Room 367, Riverside, CA 92521-0304. E-mail: jakllari@cs.ucr.edu.

- L. Tassiulas is with the Computer Engineering and Telecommunications Department, University of Thessaly, Glavani 37, 38221, Volos, Greece. E-mail:leandros@uth.gr.

Manuscript received 29 Aug. 2006; revised 16 Jan. 2007; accepted 9 Apr. 2007; published online 5 June 2007.

For information on obtaining reprints of this article, please send e-mail to: tmc@computer.org, and reference IEEECS Log Number TMC-0222-0806.

Digital Object Identifier no. 10.1109/TMC.2007.70705. certain directions. As they incorporate these main characteristics, directional antennas constitute an attractive component for all wireless devices.

Directional antennas can have many benefits in ad hoc networks. The targeted nature of the transmission results in spatial reuse, as there can be multiple transmissions in the same neighborhood without the destruction of the transmitted packets. Additionally, the directional transmission increases the signal energy toward the direction of the receiver, resulting in the increase of the coverage area. These two benefits widely lead in the increase of the channel capacity.

Unfortunately, in an ad hoc environment, directional transmissions cause some serious problems, which include increase occurrences of the hidden terminal, deafness, and the determination of neighbors' locations. The first two problems are studied extensively in [4]. The third is a natural problem that arises from the fact that, for the transmission of a packet, the transmitter must know the location of the receiver to turn over the beam to the appropriate direction.

Traditional MAC protocols [11], [12] that have been designed for ad hoc networks with omnidirectional antennas are not suitable for the support of this new feature. For that reason, there is a strong demand for the design of new protocols that will maximize the efficiency of the benefits and minimize the effects of the implied weaknesses.

In this work, we design and evaluate a MAC protocol that solves the fundamental issues arising from the deployment of directional antennas with an integrated approach. The protocol incorporates an efficient mechanism that guarantees neighbor discovery and tracking. Furthermore, by a novel use of the caches (to be described later), 
which each neighbor maintains, our protocol dramatically reduces the deafness problem and the collisions due to the asymmetry in gain. Our extensive simulations show an increase in throughput of more than 90 percent in static, as well as in mobile, topologies over previously proposed protocols and IEEE 802.11.

The rest of this paper is organized as follows: In the next section, we discuss previous related work. In Section 3, we give a summary of 802.11 and our antenna model. We propose our MAC protocol that adapts directional antennas in ad hoc networks in Section 4. In Section 5, we compare the performance of our protocol with other MAC protocols through simulation studies. Finally, in Section 6, we give the conclusions of our work.

\section{Related Work}

The idea of using directional antennas in radio communications has inspired many researchers. Zander [24] has proposed to use directional antennas in slotted ALOHA multihop packet radio networks. Some other approaches of using directional antennas have focused on broadband and cellular networks [1], [10], [23].

More recently, a few proposals have explored the required modifications in the MAC protocol of 802.11 wireless networks in order to adapt this kind of antenna in 802.11 ad hoc networks. These proposals use combinations of directional and omnidirectional transmissions for the four-way handshake frames. Nasipuri et al. [16] propose a variation of the Request To Send/Clear To Send (RTS/CTS) mechanism of IEEE 802.11 adapted for use with directional antennas. Their protocol sends the RTS and CTS packets omnidirectionally in order to enable the transmitter and receiver to locate each other and then sends the data packet and acknowledgment (ACK) in direct mode. Ko et al. [13] propose another MAC protocol that sends a directional RTS that is followed by an omni-CTS. They assume that the transmitter knows the receiver's location, so it directionally transmits the RTS to it. They propose an alternative scheme in case there is a lack of information for the location of the receiver. In this case, the RTS is transmitted in omnimode in order to seek the receiver.

Takai et al. [21] propose Directional Virtual Carrier Sensing, in which they use directional RTS and CTS transmission. For the operation of this scheme, they assume that the receiver's location is known by the transmitter. If this is not the case, they propose the omnidirectional transmission of RTS. They also propose a cache scheme where they maintain information about the location of their neighbors, which is updated every time a node receives a frame. Choudhury et al. [4] present a study about the problems that appear using directional antennas, and they propose a MAC protocol for multihop transmissions that uses a directional multihop RTS. Finally, in [14], we propose a MAC protocol for full exploitation of directional antennas in wireless local area networks (LANs). Our protocol achieves high performance using only directional transmissions and a simple scheme of tracking neighbors' directions.

Ramanathan [20] studies some interesting issues about the performance of ad hoc networks with directional antennas and the factors that affect it. He simulates several

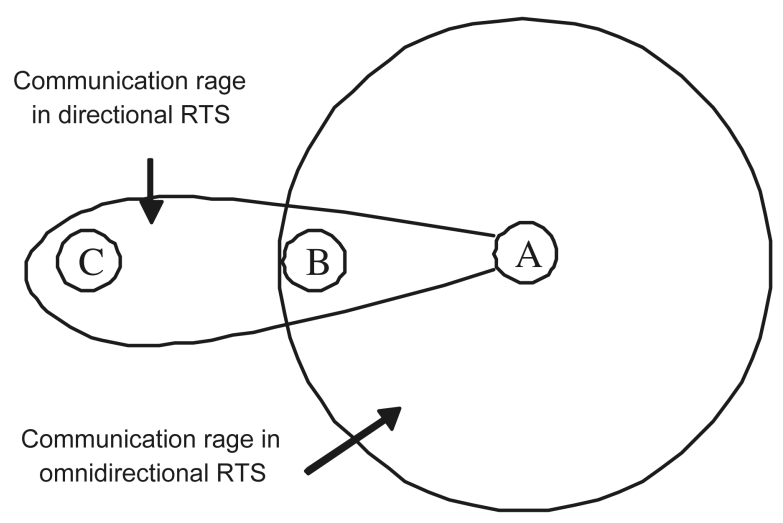

Fig. 1. The coverage range of a MAC protocol that uses an omnidirectional RTS transmission and directional CTS, DATA, and ACK transmissions.

schemes, giving useful results about the effectiveness for each one of them. ElBatt et al. [7] investigate the trade-off between spatial reuse and packet collision due to the use of directional antennas in MAC protocols. Yi et al. [22] extend the Gupta and Kumar work on the capacity of ad hoc networks and study the levels of improvement with the use of directional antennas. Choudhury and Vaidya [3] propose a new protocol, the tone-based directional MAC (ToneD$\mathrm{MAC}$ ), which is a tone-based mechanism for addressing the deafness problem. They consider one-hop and multihop scenarios. Finally, in [2], they advocate the use of directional antennas on ad hoc routing protocols.

The previous studies have some common characteristics that lead to inefficiencies of the proposed protocols. Papers [16], [13], and [20] employ at least one omnidirectional transmission of a control packet, limiting in this way the coverage area. If there is even one omnidirectional frame in the handshake between the transmitter and receiver, then the directional transmissions must be limited in the coverage of the omnirange as the maximum distance between the two stations is defined by the smaller coverage range of the four frames of the handshake. Assume that the RTS transmission is in omnimode, whereas the other three transmissions (CTS, DATA, and ACK) are in directional mode. Then, the directional mode transmissions must reduce their transmission energy to cover no more than the coverage area of the RTS. This is clearly depicted in Fig. 1 and constitutes a weakness of the mentioned MAC protocols as, in this way, they do not exploit one of the main benefits of the directional transmission, the increase of the coverage range.

As described in [5], [15], and [8], with $M$ antenna elements and with the same transmission energy, an antenna array provides an increased antenna gain in comparison with the omnimode of the order of $M$. Roughly speaking, this gain is doubled if there is directivity in both transmission and reception. Therefore, a directional communication between two stations can have a significant increase in the distance between them compared with the equivalent omnicommunication. This benefit is not fully exploited by the above schemes.

In [4] and [21], the use of only directional transmissions in the four-way handshake does not overcome the main 
problems: the increase of instances of the hidden-terminal problem, the problem of deafness, and the problem of determination of neighbors' locations. The reasons why the first two problems arise are studied explicitly in [4]. The third problem arises from the fact that sending a directed RTS assumes that the information about the receiver's location is known or is given by another mechanism or from the above layer. This assumption greatly simplifies the scheme, making it unsuitable for implementation without the contribution of an external mechanism, the design of which is not itself a trivial problem.

To be more accurate, the important information about the location of a node is not the location in its own right, but the direction the node must turn its beam in order to have access to its neighbor. Therefore, the knowledge of the position of a node (which Ko et al. [13] assume by means of additional hardware such as GPS) may not always be the right guide for the decision of the beam direction. A physical obstacle between the receiver and transmitter can exist, so the connectivity may occur by reflection of the radio wave, resulting in signal reception from a direction different from the neighbor's direction.

In [4], [13], and [21], the authors discuss the concept of a directional Network Allocation Vector (D-NAV) that adapts the concept of NAV as is introduced by IEEE 802.11 into the directional environment. These studies contain an interesting qualitative description of the D-NAV without the functional details that must be defined to make its use beneficial.

We propose a MAC protocol that uses only directional transmissions and a simple scheme of tracking neighbors' directions. This achieves high performance while minimizing the hidden-terminal problem due to asymmetry in gain, as well as the deafness problem. The innovative scheme of tracking neighbors' directions efficiently solves the problem of defining the neighbor's location, making the proposed protocol implementable in an environment without prior knowledge of the nodes location. In [14], we have given the basic description of our protocol and some first numerical results, which show its efficiency in static scenarios. In this paper, we present extended simulation experiments that clearly show the significant performance improvement of the protocol in static, as well as in mobile, scenarios.

\section{Preliminaries}

\subsection{IEEE $\mathbf{8 0 2 . 1 1}$}

We summarize some attributes of IEEE 802.11 [11], [17] that are important for its operation with directional antennas. In the case of MAC in ad hoc networks, 802.11 introduces a Distributed Coordination Function (DCF) that coordinates the access using a carrier sense multiple access with collision avoidance (CSMA/CA). A station must listen to the medium, and if it finds it idle for a predefined time, (DFTS) it can start data transmission to the receiver. When the receiver listens to the data packet, it responds with an ACK packet.

To deal with the hidden-terminal problem [9], [6], an enhancement of the scheme that uses a handshake of RTS and CTS frames can be added to the basic transmission scheme (Data+ACK). This scheme guarantees that the receiver is in a state that is capable of receiving a data packet. The transmitter informs the receiver of the intended transmission. If it receives a CTS immediately after its RTS, it starts the transmission of data; otherwise, it backs off. In this way, a four-way handshake is created (RTS-CTSDATA-ACK) that is used for collision avoidance.

The first three frames of the handshake (RTS-CTSDATA) contain information about the duration of the pending handshake, informing the neighbors to avoid starting a new transmission during this period. This is managed by a mechanism called Virtual Carrier Sense. In this mechanism, every station maintains a $N A V$. If NAV is equal to zero, the station can transmit; otherwise, it cannot. $\mathrm{NAV}$ is initially equal to zero. If a NAV is a positive number, there is a countdown until it reaches zero. When a station listens to one of the four frames, it updates its NAV with the duration of the pending handshake, preventing itself by transmitting until its NAV reaches zero again. With this scheme, every station performs a Virtual Carrier Sense in addition to the physical carrier sense to enhance the resistance of the protocol against collisions.

\subsection{Directional Antennas}

A directional antenna can transmit a signal in any direction, using an array of antennas called array of elements. Individual omnidirectional transmissions from these elements interfere positively or negatively with each other, resulting in an increase of signal strength in one or more directions and elimination in others. The greater the number of elements of a directional antenna, the better the increase of the signal in the desired direction. There are directional antennas with one (omnidirectional), two, four, eight, 16, and so forth elements. The interested reader can find an intensive study of directional antennas in [15] and [19].

As the number of antenna elements increase, the beam width and the signal gain can be controlled more effectively. An important element of our protocol is covering the whole area around the transmitter with successive sequential transmissions. In the rest of the paper, we will assume that we can provide effective omnitransmission with $M$ sequential directional transmissions when we have $M$ antenna elements. In certain cases, this number may need to be higher, but our conclusions, which are based on the numerical experiments we performed, will not change.

As we can see in Fig. 2, the area around the node is covered by $M$ beams. We assume that the beams are not overlapping. We number the beams from 1 to $\mathrm{M}$ starting from the beam that is located just right of the three o'clock position. The node can transmit its signal to any one of the $M$ beams, increasing the coverage range of the transmission toward a specific direction. In idle mode, the node listens omnidirectionally. In the reception of a signal, the node uses selection diversity, which means that it uses the signal from the antenna that is receiving the maximum power of the desired signal. With this mechanism, the receiver can extend the communication area. Therefore, the communication link can benefit more by beamforming at both the transmitter and receiver. 


\section{The Proposed Protocol}

In the previous section, we summarized the advantages, disadvantages, and functional characteristics that appear in ad hoc networks when directional transmission is used. These points can constitute a guideline for the principles that must govern the design of a MAC protocol in order to coordinate the communication in these environments. We are going to outline these principles in the following lines:

- An effective protocol must use only directional transmissions to fully exploit the increase in coverage range with the use of directional antennas.

- There must be a mechanism that informs the neighbors to defer their transmission if this is going to harm the pending transmission, thereby decreasing the problem of a hidden terminal.

- There must be a mechanism to deal with the problem of deafness.

- The proposed scheme must provide an efficient way to identify the neighbors' locations and to maintain this information for use in directional transmissions. This scheme must be simple and must exchange very short information between stations if this is necessary.

Proposed MAC protocols that use at least one omnidirectional control frame transmission [13], [16], [21] do not comply with the first principle, but they comply, in a sense, with the second and third principle. This happens due to the fact that the omnitransmission of the control frame spreads around the information about intended transmissions. Regarding the fourth principle, although [13] and [21] propose schemes for the solution of the neighbors' location problem, they do not give a detailed description. On the other hand, a part of [21] and the proposed MAC protocol in [4] observe the first principle, as they propose the use of directional transmissions for RTS-CTS, but they do not deal with the problems of a hidden terminal and deafness as it is described in detail in [4]. Furthermore, in [4], Choudhury et al. assume the providence of neighbors' location information by an upper layer resulting in the lack of observation of the fourth principle.

In the following paragraphs, we are going to describe our proposed protocol, which is named Circular Directional RTS MAC (CDR-MAC). Our protocol has been designed under the guidance of the principles that we have just mentioned. It is simple in the implementation, as it is based on the concept of the IEEE 802.11 protocol. Nevertheless, it uses only directional transmissions to increase the coverage area. Moreover, the transmitter informs the neighbors to defer their intended transmission if it is going to harm the initial one. Finally, our proposal neither assumes a priori any information about the neighbors' locations nor depends on any information that it receives for that reason from an upper layer as in [4]. It provides a simple scheme for the recording and maintenance of a neighbor's location, and the information exchanging between neighbors is minimal. Furthermore, this scheme assists the functionality of the D-NAV scheme proposed in [13], making it simpler in implementation.

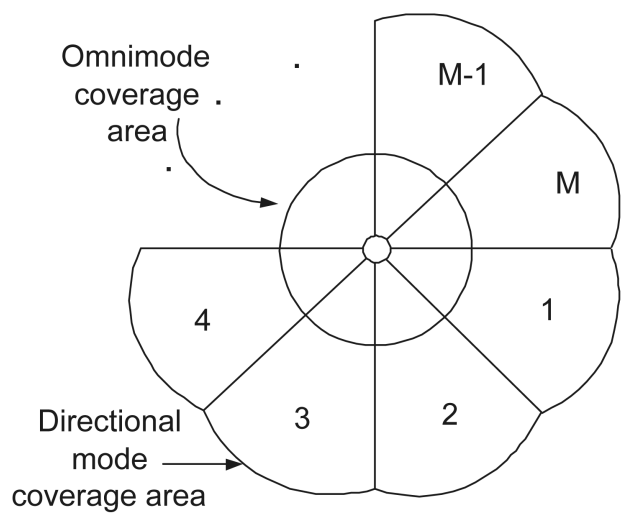

Fig. 2. A node with $M$ beams.

\subsection{Circular Directional RTS}

Our protocol is based in a simple and innovative scheme of RTS transmission. In this scheme, the RTS is transmitted directionally consecutively in a circular way until it scans all the area around the transmitter. The circular transmission of the CTS is an alternative that may enhance the performance of the protocol as well. Since this may result in the unpredictable behavior of the communicating stations, we will not study this factor in this work. We are going to investigate this idea in our future studies.

As mentioned, we assume antennas with a predefined number of beams, $\mathrm{M}$ in Fig. 2, which cover the area around the transmitter. Assume that, with beam 1, the transmitter starts transmitting its RTS in a predefined direction. Shortly afterward, it turns its transmission beam on the right, sending the same RTS with the next one (beam 2). It continues this procedure again and again until the transmission of RTS covers the entire area around the transmitter (until it sends the RTS with beam M).

The RTS contains the duration of the intended four-way handshake (as in 802.11). As this information is spread around by the circular RTS, the neighbors are informed about the intended transmission. The neighbors, after executing a simple algorithm that is described later in this section, decide if this harms the ongoing transmission whether they will defer their transmission in the direction of the transmitter or receiver. In this way, the neighbors are aware of the intended handshake, a fact that results in reduction of the hidden-terminal problem.

The station that is the destination of the RTS waits until the circular RTS transmission is finished, and afterward, it sends a directional CTS toward the direction of the transmitter of the RTS. On the other hand, when the transmitter completes the circular transmission of RTS, it listens to the medium omnidirectionally to receive this CTS. That means that the carrier sensing from the transmitter of the RTS in this phase is performed in an omnidirectional mode, just as in 802.11. If the CTS is received during a predefined period (CTS time out), then the transmitter continues with the transmission of the data packet and the reception of the ACK, just as in 802.11, except for the fact that now the transmissions of the data and ACK packets are directional. By using only directional transmissions of RTS, CTS, DATA, and ACK, we exploit the benefit of increasing 
TABLE 1

A Record of the Location Table Maintained by Node A in Fig. 3

\begin{tabular}{|c|c|c|c|}
\hline Me & Neighbor & My Beam & Neighbor's Beam \\
\hline A & B & 4 & 2 \\
\hline
\end{tabular}

coverage area, compared with the case of at least one omnidirectional transmission that limits the coverage area in this of omnimode transmission.

It is important to mention that the transmitter and the receiver do not need any information about each other's location. The circular RTS reaches the target node wherever it is located. On the other hand, the receiver, using selection diversity, receives the signal from the right beam and so sends its CTS by the same beam. Nevertheless, we propose a simple scheme for the tracking and maintenance of other nodes' locations, which helps for a more efficient functionality of the network.

\subsection{Neighbors' Locations}

Due to the lack of location information, the transmission of the RTS is a difficult issue in the directional transmission of the four frames. If the receiver starts receiving the RTS, using selection diversity, it will recognize the direction by which it receives the signal, and so, it will transmit the CTS in the right direction. Accordingly, the transmitter recognizes the direction of the receiver by the reception of the CTS and sends the data packet in the right way. Our protocol guarantees the transmission of the RTS in the direction of the receiver by the CDR. Therefore, if the handshake is completed, the transmitter and receiver will know each other's relative directions. More precisely, each of them knows the beam by which it can reach the other. Our protocol exploits this information by a simple scheme to solve the nodes location problem.

According to that scheme, every node maintains a table, called Location Table, with one record for every station that it has heard. Initially, the Location Table is empty, and it is updated in every reception. Because of the mobility of the stations, a record may be updated many times.

In every record, the node maintains the following information: $M e$ (itself), Neighbor (the station from which it has received a packet), My beam (the beam from which the transmitter received the packet), and Neighbor's beam (the beam by which the receiver sent the packet). In this way, every station maintains pairs of beams that are used for a direct transmission.

The record in Table 1 means that $\mathrm{A}$ can transmit or receive from $B$ by beam 4 , whereas $B$ can transmit or receive from A by beam 2. This is illustrated in Fig. 3, where every station uses four beams. The information " $\mathrm{A}, 4$ " is known from A by the selection diversity mechanism. The problem is that A cannot realize the beam by which $\mathrm{B}$ receives its packet. For that reason, in every packet, in addition to the other information, the transmitter sends a number that indicates the beam by which the packet is transmitted. In this way, by the reception of a frame, the receiver can update all the information of the corresponding record.

As we have mentioned, this information is not necessary for a four-way handshake communication to take place due

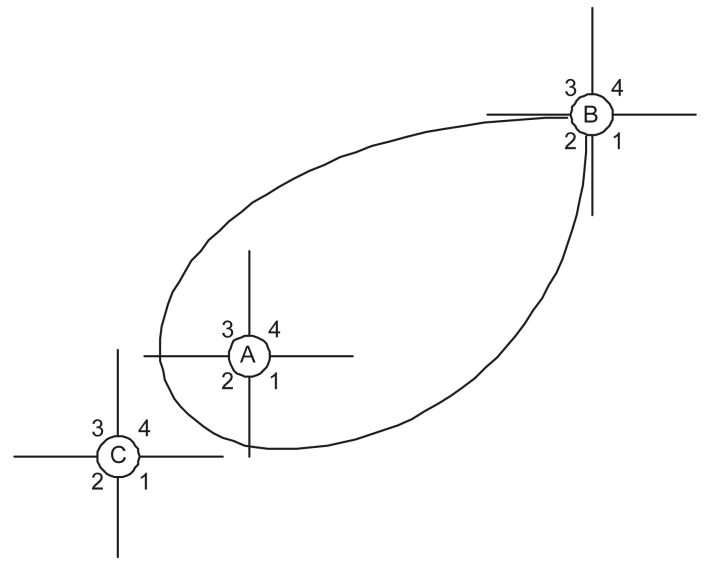

Fig. 3. An example scenario of the hidden-terminal problem.

to the CDR. Nevertheless, this information is useful in the decision of the neighbors about the deferment of their transmission or not, as we explain in the next section.

\subsection{Use of D-NAV}

As we have discussed, one of the main problems of the directional transmissions in ad hoc networks is the increase of the hidden-terminal problem. Our protocol deals with this problem by informing the neighbors of the intended transmission. The neighbors receiving the circular RTS (or, alternately, the CTS) have to decide if it is necessary to defer their transmission in any specific location. In [21] and [4], there is an extensive discussion about this problem. Both of these papers adopt the $D-N A V$ scheme proposed in [13] as a mechanism to deal with this issue. D-NAV functions in the philosophy of the NAV proposed by IEEE 802.11, adapted in the directional communication.

D-NAV uses a table that keeps track of the directions and the corresponding durations toward which the station must not initiate a transmission [4]. In order to keep neighbors silenced toward the right direction during a transmission, the continuous update of this table with the right information is important both for dealing with the hidden-terminal problem and for spatial reuse.

The right update of the D-NAV table has two issues to address. The first is informing the neighbors about the intended transmission. The second is the right knowledge of neighbors of transmitter's and receiver's locations for the proper decision about the directions in which a neighbor must defer its transmission in order not to destroy the intended handshake.

Papers [21] and [4] qualitatively discuss that issue, but they do not propose a practical algorithm for the right update of the D-NAV table. By assuming the knowledge of a node for other nodes' locations, Choudhury et al. [4] greatly simplify the problem, making it trivial. Moreover, the directional transmissions decrease the spread of the information that is given by the RTS-CTS exchange about the intended transmission.

In [21], Takai et al. propose setting D-NAV toward the location of the station by which it receives the RTS, CTS, or data packet. In that way, a neighbor cannot realize by the reception of RTS the location of the intended receiver of 


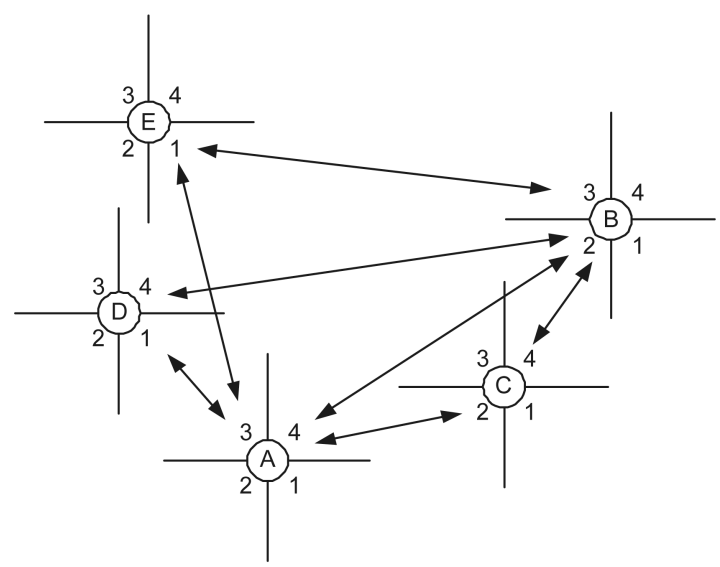

Fig. 4. An example scenario where nodes use their Location Tables.

the RTS. Therefore, it can only defer toward the location of the transmitter. Consequently, for the right update of the D-NAV in an intended transmission, a neighbor must receive both RTS and CTS packets. Otherwise, it will update its D-NAV table only toward one of the two nodes, and so it may destroy the transmission. (Please bear in mind that, for a successful transmission, there must be no collision in the receiver, as well as in the transmitter of the data packet, because of the exchange of the handshake).

One other problem that is discussed in [4] is the hiddenterminal problem due to asymmetry in gain. This problem is presented in Fig. 3. Here, node A sends RTS to B, and C is a neighbor. When $A$ and $C$ are in idle mode, they listen to the medium omnidirectionally. As we can see, B's beam can just reach $A$, making $C$ a hidden node for $B$. In this scenario, $C$ is not able to receive A's RTS or B's CTS. Therefore, C is not aware of the transmission of the data frame from $A$ to $B$. When A starts transmitting the data frame directly to B, B, using selection diversity, receives directionally too. If $C$ sends a frame toward B's location, while this transmission is in progress, $\mathrm{B}$ can receive this frame due to its directional reception, confusing the two signals. This confusion results in packet collision.

Our protocol solves these problems with a very simple mechanism. As we have mentioned, every station maintains a Location Table. In this table, pairs of antenna beams are kept to interact for the communication between itself and its neighbors. When a station transmits an RTS or CTS to another station, the frame header contains the corresponding beam pair. Every neighbor that receives one of these frames examines its Location Table to find the beams through which it can "see" the two stations. If one of these beams coincides with the respective beam of the RTS and CTS frames, then the neighbor defers its transmission by this beam. Let us see this mechanism by the example illustrated in Fig. 4.

In this figure, station A starts a transmission for station B. Stations $C, D$, and $E$ are neighbors that receive the circular RTS from A. The RTS as we can conclude by the figure will contain the following information: A, B, 4, and 2. That means that $\mathrm{A}$ is going to send (RTS, DATA) and receive (CTS, ACK) frames by beam 4, whereas $B$ is going to send (CTS, ACK) and receive (RTS, DATA) frames by beam 2 . In
TABLE 2

Modules of Location Tables Maintained by Nodes C, D, E in Fig. 4

\section{For Node C}

\begin{tabular}{|c|c|c|c|}
\hline Me & Neighbor & My Beam & Neighbor's Beam \\
\hline C & A & 2 & 4 \\
\hline C & B & 4 & 2 \\
\hline
\end{tabular}

For Node D

\begin{tabular}{|c|c|c|c|}
\hline Me & Neighbor & My Beam & Neighbor's Beam \\
\hline D & A & 1 & 3 \\
\hline D & B & 4 & 2 \\
\hline
\end{tabular}

For Node E

\begin{tabular}{|c|c|c|c|}
\hline Me & Neighbor & My Beam & Neighbor's Beam \\
\hline E & A & 1 & 3 \\
\hline E & B & 1 & 3 \\
\hline
\end{tabular}

the figure, we can see that the corresponding modules of the Location Tables in the three nodes are as depicted in Table 2.

By the information that is contained in the RTS, the three neighbors know that $\mathrm{A}$ will receive by beam 4, whereas B will receive by beam 2 . Hence, every neighbor examines its Location Table (illustrated in Table 2) to see if it can destroy the reception in A or B. If this occurs, it defers its transmission by the corresponding beam updating its D-NAV table.

Let us see how $\mathrm{C}, \mathrm{D}$, and $\mathrm{E}$ will act in the previous scenario. $C$ recognizes that $\mathrm{A}$ can receive it by beam 4 , whereas $B$ can receive it by beam 2 . Thereby, it realizes that it can interfere in the reception of both $\mathrm{A}$ and $\mathrm{B}$. This information leads $C$ to defer its transmission for the corresponding duration by the beams that can transmit this interference, namely, beams 2 and 4, updating the D-NAV table.

D will recognize that $A$ can receive it by beam 3 , whereas $B$ can receive it by beam 2 . Thereby, it realizes that it can interfere only with the reception of $\mathrm{B}$, and so, it defers its transmission through beam 4, updating its D-NAV table.

Accordingly, E realizes that it cannot harm the intended transmission, and so, it does not defer its transmission by any beam.

It is worth noting that the three nodes act in that way having received only RTS from A. Due to the lack of the extra information that we have added in RTS, in previously proposed protocols, neighbors receiving RTS cannot realize the beam by which $B$ will receive the data frame. Therefore, they will update their D-NAV table only with the information about the location of $\mathrm{A}$, not protecting in that way the reception in $\mathrm{B}$. They must also receive CTS in order to realize B's location and, hence, to protect the reception in this node.

Let us see how our protocol will affect the collision in Fig. 3. Node $\mathrm{C}$ will receive the CDR from $\mathrm{A}$, being aware for the intended transmission. Reading the extra information into RTS about the beams that will be used for this 
transmission, $\mathrm{C}$ will examine its location table and will defer the transmission toward B's location (that is, beam 4), resulting in this way in the avoidance of the collision.

Our protocol supports in an efficient way the mobility of the stations, as the Location Table is updated very often (every time a node receives a packet), leading to a contentious tracing of neighbors positions.

\subsection{Some Protocol Details}

There are some details related to the implementation of the protocol. These are the necessary changes in some time periods that have been defined in 802.11 in order to support our protocol. In the next paragraphs, assume that $\mathrm{A}$ is the transmitter node and $\mathrm{B}$ is the receiver.

1. CTS Is Transmitted after the Circular RTS.

Now, the CTS frame must be transmitted by B just after the conclusion of the circular RTS. For this reason, B waits for time $\mathrm{T}$ (instead of Short Interframe Space (SIFS) in 802.11) after the reception of RTS and then transmits the CTS. The time T is calculated as follows:

$T=k{ }^{*} R T S$ transmission time $+S I F S$, where $k=$ $M-A^{\prime}$ 's beam number.

Thus, if the B receives an RTS by A by its first beam (beam 1) and $\mathrm{M}=4$, it will wait for $(4-1)^{*} R T S$ transmission + SIFS. This is the time needed for three more RTS transmissions until the conclusion of the circular RTS transmission. If B receives an RTS from A by its final beam (beam 4), then it will send CTS SIFS time after RTS reception (as now $\mathrm{k}$ is 0 ).

During the waiting time T, B is locked in a "readfor-transmission" mode, ignoring the reception of other packets.

2. An Idle Node Listens to the Channel for a Longer Time than DIFS before Transmission.

It is obvious that now every idle node must listen to the channel more than DIFS before its transmission, as now RTS is circular, and so its spread around a node takes more time compared with that when in omnimode. For that reason, in our simulations, we defined an idle node to listen to the medium for $M^{*} R T S$ transmission time before its transmission.

3. Duration Field of the RTS Packet Is Decreased by the RTS Transmission Period Every Time an RTS Frame Is Transmitted in the Cycle.

As we have mentioned, RTS informs neighbors about the intended data transmission. In 802.11, the RTS duration field contains the time period that is needed for the conclusion of the whole handshake. Thus,

$$
\begin{aligned}
\text { Duration period }= & \text { RTS trans time }+ \text { SIFS } \\
& + \text { CTS trans. Time }+ \text { SIFS } \\
& + \text { Data trans time }+ \text { SIFS } \\
& + \text { Ack trans time. }
\end{aligned}
$$

Now, the RTS transmission time depends on the beam that is to be sent. Thus,
For the first beam: Circular RTS trans time $=$ $(M-1)^{*} R T S$ trans. time,

For the second beam: Circular RTS trans time $=$ $(M-2)^{*} R T S$ trans. time, and so forth.

4. The Circular RTS Respects the Ongoing Transmissions.

If the D-NAV of A does not allow the transmission of the RTS toward a specific direction, A does not send the RTS toward this direction, remaining in silent mode during the corresponding period. In this way, the transmitter respects the ongoing transmission.

\section{Performance Evaluation}

The performance evaluation was performed in OPNET Version 10.0 [18]. We chose the specific tool because it offers very good support for simulating directional antennas. The antenna editor tool of OPNET supports the creation of arbitrary $3 \mathrm{D}$ gain patterns. The main beam can be aimed at any arbitrary point in 3D space, and the energy received at every node is computed automatically by OPNET kernel procedures. Using OPNET for the experiments allows us to deploy more realistic antenna models. Except for static scenarios, we consider scenarios with mobile users as well. Thus, we present interesting results about the efficiency of the protocol in a dynamic environment where the behavior of the stations is more unstable.

In our simulations, we assumed that the physical channel is error free and the propagation delay is zero. The destination of each packet is chosen randomly from the set of the station's neighbors. The packet length is constant and equal to 1,024 bytes. The packet arrival at each station is a Poisson process with the same mean $\lambda$. To vary the load of the network, the $\lambda$ is varied. The value of $\lambda$ depends on the number of stations that participate in the scenario and the overall load we want to achieve.

The performance metric used to evaluate the protocols is the aggregate throughput achieved by the network. As throughput, we define the percentage of the channel rate that is used for the transmission of data packets. An overall throughput that exceeds 100 percent means that there is more than one pair of nodes that communicate simultaneously for a period of time. Therefore, such a result is an indication of the average channel reuse ratio due to the use of directional transmissions.

Each simulation runs for 200 seconds with a warm up period of 50 seconds. In our simulations, we have used nodes that were equipped with antenna arrays of one, four, and eight elements. In the start of every simulation, the Location Table of each station is empty and is gradually updated as the simulation progresses.

In our simulations, we study the benefits of our protocol, comparing it with other MAC protocols with omnidirectional and directional transmissions. More particularly, we compared it with 802.11 (which uses omnitransmissions), as well as with a directional MAC (D-MAC) protocol that uses directional RTS and CTS transmissions, as this is described in [4]. We tried to ensure that the scenarios we have chosen guarantee equal conditions between the examined protocols. As a result, we simulate our protocol with D-MAC in 


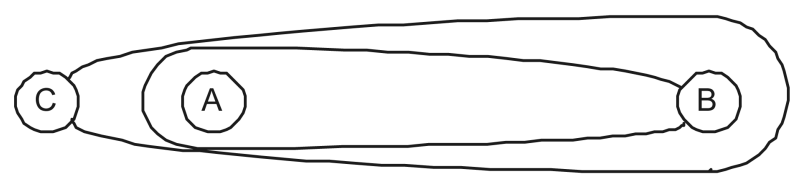

Fig. 5. The transmissions of the scenario in Fig. 3.

specific scenarios with known topologies, as D-MAC assumes the knowledge of the location of the neighbors by any node. It makes no sense to compare these two protocols under lack of locations' knowledge conditions, as D-MAC would not operate properly. As the proposed protocol is the only integrated solution that uses directional transmissions and can operate without any assumptions about neighbors' location, we compare it in arbitrary scenarios, where nodes have no knowledge of their neighbors' locations, only with 802.11.

\subsection{Static Scenarios}

We first evaluate the performance of the scenario demonstrated in Fig. 3. We have selected this simple scenario to compare the effectiveness of our protocol with that of the D-MAC [13], which uses directional RTS-CTS. As we can see in Fig. 5, A transmits to B, whereas $C$ transmits to A. The coverage range of B's CTS does not include $C$. On the other hand, as B receives directionally toward A's direction, it receives the packets that are simultaneously transmitted by $\mathrm{A}$ and $\mathrm{C}$, a fact that leads to collision. The results are shown in Table 3.

As we have discussed in the previous section, D-MAC comes short in throughput because of the hidden-terminal problem due to the asymmetry in gain. Our protocol overcomes this problem, as $\mathrm{A}$ informs $\mathrm{C}$ of the intended transmissions by its circular RTS resulting in a throughput that is double the throughput of D-MAC.

In Table 3, we can also see that D-MAC has a fairness problem. Node $\mathrm{C}$ has almost half of the throughput of Node A. On the other hand, in our protocol, the overall throughput is shared equally between the two nodes (around 40 percent for each one). This happens due to the fact that, in D-MAC, node $C$ cannot be aware of the communication between A and B (as the RTS from A is directional toward B and the CTS from B cannot reach C), so assuming that the medium is idle, it sends RTS again and again. As it does not receive a response from A (A is communicating with B), it backs off again and again, resulting in this way in large back-off periods. Our protocol provides to $\mathrm{C}$ the information for the ongoing communication (by the circular RTS), resulting in the avoidance of these large back-off periods.

TABLE 3

Simulation Results of the Scenario in Fig. 3 that Study the Hidden-Terminal Problem Due to Asymmetry in Gain

\begin{tabular}{|c|c|c|}
\hline $\begin{array}{c}\text { Throughput (\%) } \\
\text { in high load }\end{array}$ & D-MAC & Our protocol \\
\hline Node A & 33.34 & 40.21 \\
\hline Node C & 15.57 & 39.89 \\
\hline Overall & $\mathbf{4 8 . 9 1}$ & $\mathbf{8 0 . 1}$ \\
\hline
\end{tabular}

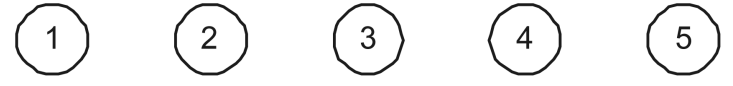

Fig. 6. A scenario with linear topology.

Our second experiment examines the overhead that is added by the circular transmission of the RTS. In the proposed protocol, the transmission of the RTS lasts longer than in the directional mode or omnimode due to the fact that now RTS is transmitted M times.

By simulating the scenario in Fig. 6, we measure the throughput degradation of our protocol in comparison with the directional transmission of RTS-CTS in a case where our protocol does not benefit by its mechanism. In this scenario, as all nodes are in a linear topology, the transmission of the circular RTS is not necessary, as now all nodes can receive the directional RTS-CTS, and so they can be informed of the intended transmissions. To clearly see the effect of the circular RTS, we enhanced the directional RTS-CTS with the feature of informing the neighbors of the two stations that participate in the communication, just as in our protocol.

Table 4 shows the results of the simulation. As we can see, the proposed protocol has a degraded throughput of 3.5 percent in the case of $M=4$ and 7 percent for $M=8$. This overhead is due to the repeated transmissions of the RTS and is quite low in comparison with the benefits that arise by its use.

In our next experiment, we evaluate the throughput performance of a scenario with seven nodes randomly distributed in a $2 \mathrm{D}$ area. We examine the performance of our protocol compared with that of 802.11, as the offered load increases. We assume that every node can listen to the others and that the coverage range of the three transmission models (antennas with one, four, and eight elements) is equal. In this way, we are going to measure the effect of the circular directional transmission of the RTS, keeping the other parameters constant. Please bear in mind that, in the presented results, we must add the extra enhancement of the throughput due to the increase in the coverage range.

The variation of the total throughput of the network with the total offered load is shown in Fig. 7. As we can see, the proposed protocol highly increases the throughput as the number of antenna elements is increased. In heavy-load conditions, the throughput of our protocol has a gain of 34 percent in the case of four elements and 42 percent in the case of eight elements, compared with 802.11. In light-load conditions, our protocol seems to have a slightly lower throughput than 802.11. This happens due to the fact that,

TABLE 4

Simulation Results of the Scenario in Fig. 6 that Measure the Effect of the Circular RTS

\begin{tabular}{|c|c|c|c|}
\hline & \multirow{2}{*}{$\begin{array}{c}\text { Enhanced } \\
\text { D-MAC }\end{array}$} & \multicolumn{2}{|c|}{ Our Protocol } \\
\hline & & $M=4$ & $\mathbf{M}=\mathbf{8}$ \\
\hline $\begin{array}{l}\text { Throughput }(\%) \\
\text { in high load }\end{array}$ & 169.11 & 163.54 & 157.4 \\
\hline $\begin{array}{r}\text { Degradation (\%) } \\
\text { with Enhanced }\end{array}$ & $\begin{array}{l}\text { compared } \\
\text { D-MAC }\end{array}$ & 3.29 & 6.92 \\
\hline
\end{tabular}




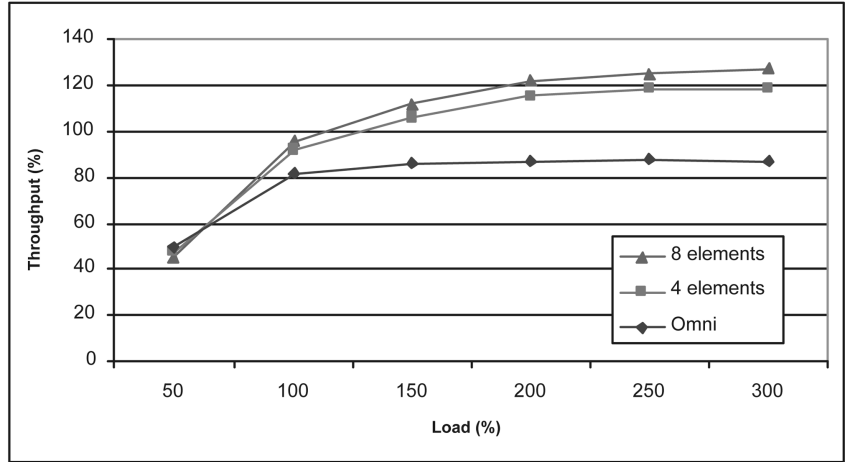

Fig. 7. Throughput of the proposed protocol using antennas of one, four, and eight elements for an arbitrary scenario of seven stations.

in such conditions, directional transmissions cannot benefit from the spatial reuse as the load is very light, whereas the circular transmission of the RTS adds an extra overhead compared with the omnitransmission in 802.11. As the load increases, the loss incurred in overhead is quickly regained by the increase of the throughput due to the spatial reuse.

Our next experiment is a generation of the previous scenario in a more arbitrary environment with more stations. We have used a topology with 15 stations randomly distributed in a $2 \mathrm{D}$ area. We have made the same assumptions again, that is, every node can listen to the others and the coverage range of the three transmission models (antennas with one, four, and eight elements) is assumed to be equal. We run the experiment for 10 different arbitrary topologies of 15 stations. In Fig. 8, we give the average results.

As we can see in Fig. 8, the picture is proportional to that of the previous scenario. What is worth mentioning is that the overall throughput of 802.11 converges again to almost the same value. This is something we expect as there is no spatial reuse in both scenarios of 802.11 because every station can listen to the others. On the other hand, the throughput of our protocol increases compared with that in the situation of the seven stations. This happens since there are more stations with this scenario, increasing the probability of simultaneous transmissions between pairs of stations, a fact that increases the spatial reuse.

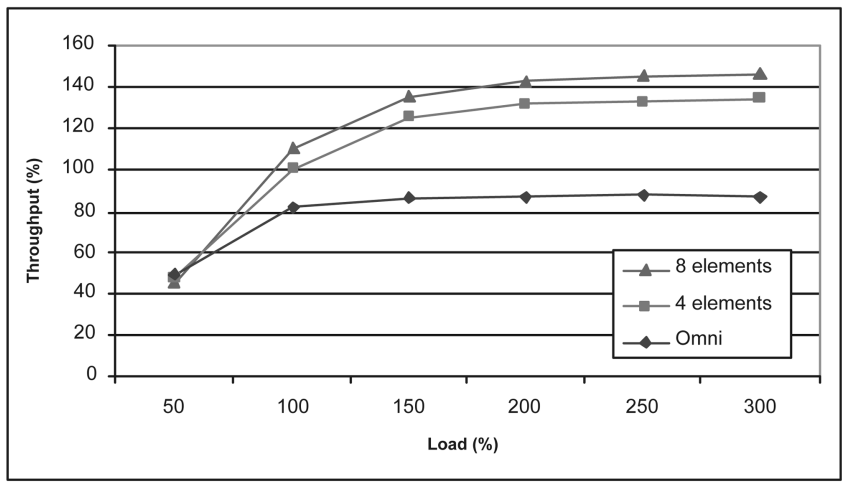

Fig. 8. Throughput of the proposed protocol using antennas of one, four, and eight elements for an arbitrary scenario of 15 stations.

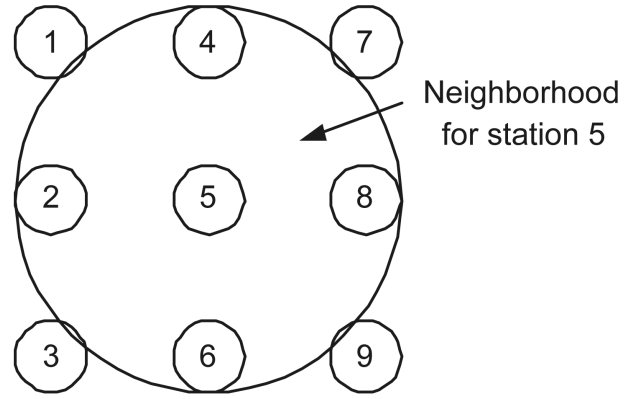

Fig. 9. A grid topology scenario.

Our final experiment uses the scenario in Fig. 9. There are nine nodes in a grid topology. The neighbors of the nodes can be concluded by the circular range around station 5 that depicts the coverage range of the eight beams of this station.

The results of the simulations are shown in Fig. 10. Our protocol performs better than 802.11 as the offered load increases. In heavy-load conditions, the throughput achieved by the proposed scheme is double the throughput of 802.11. This happens due to the fact that spatial reuse is stronger as the load increases. On the other hand, in the case of 802.11, the hidden-terminal problem and the exposedterminal problem (where there are differences in nodes that do not destroy any transmission) do not enable a high-level spatial reuse.

\subsection{Random Scenarios with Mobility}

It is very common for the nodes of a wireless LAN to be mobile. Mobility is a typical characteristic of such an environment. Thus, it is important to examine the behavior of our protocol in scenarios where the nodes are mobile. The efficient update of the Location Table and the accurate transmission of the frames toward the right direction are very important issues for the right behavior of the protocol. To study the efficiency of the protocol in a mobility environment, we consider some random scenarios. We measure the throughput in every scenario for static, as well as for mobile, nodes. We examine cases with both low and high mobility for the nodes. With low mobility, nodes are assumed to move with speeds of $2 \mathrm{~m} / \mathrm{s}$, whereas, with high mobility, they move at $10 \mathrm{~m} / \mathrm{s}$.

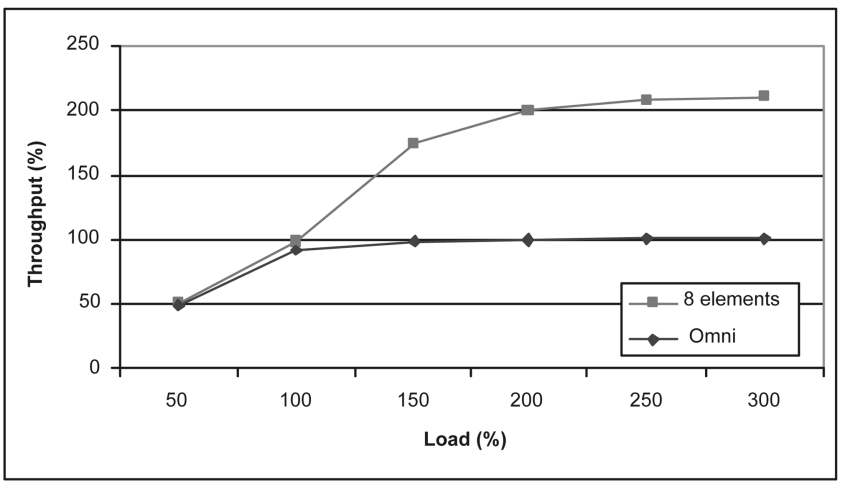

Fig. 10. Throughput of the proposed protocol for the grid topology in Fig. 9. 


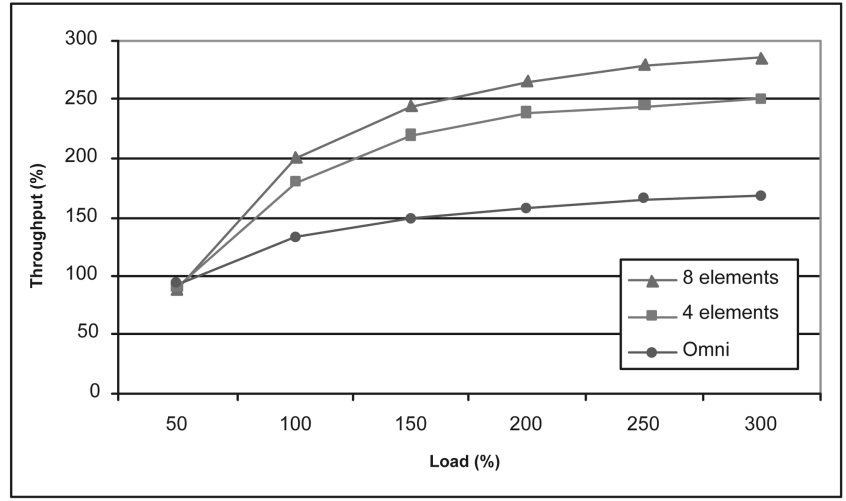

Fig. 11. Throughput for the random scenario of 60 static nodes.

We consider a random scenario that consists of 60 nodes. We assume that the nodes are located in a 1,250 $\times 1,250 \mathrm{~m}^{2}$ square area. The omnidirectional coverage range of each node is $250 \mathrm{~m}$. First, we consider the case of static nodes. We run the experiment for 10 different random topologies and we take the average results. The average throughput is depicted in Fig. 11.

As we can see from this figure, our protocol performs much better than the 802.11 in the random scenario. Similar to the arbitrary scenarios of seven and 15 nodes in the previous section, the throughput of our protocol increases as the load of the nodes increases. The throughput also increases as the number of elements per node increases. We must notice that, in this scenario, where the nodes are spread in an area that is quite large compared to the coverage area of a node, there is spatial reuse even in the case of the omnidirectional transmission. Thus, the throughput of this case in heavy-load conditions is also more than 100 percent of the offered load.

We again run the same random scenario with 60 nodes, but now, the nodes are mobile. The mobility of the nodes is low. We examine the effect of mobility in the efficiency of the protocol. The throughput in comparison with that in the static scenario is depicted in Fig. 12.

In this figure, we can see that our protocol behaves efficiently in the mobility environment. There is a slight decrease in the throughput in the case of mobile users compared with that of static users. This is due to the following facts: When nodes are mobile, there is a

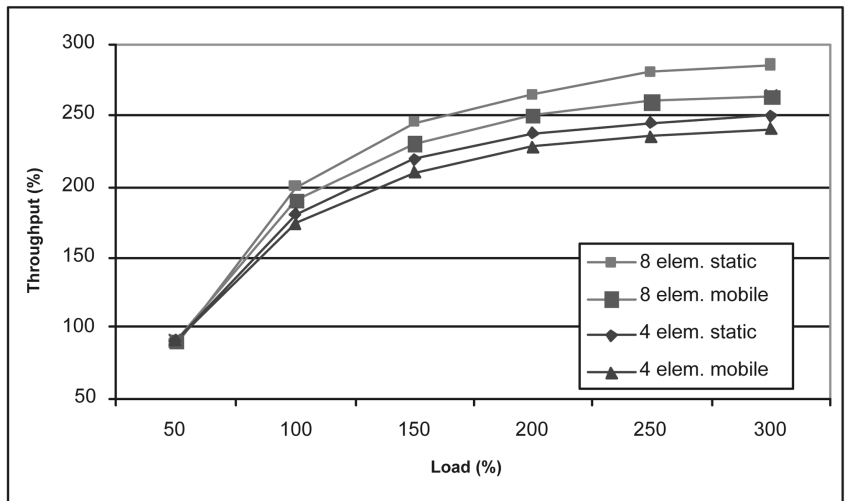

Fig. 12. Throughput for the random scenario of 60 mobile nodes.
TABLE 5

Throughput Degradation for the Random Scenario of 60 Mobile Stations

\begin{tabular}{|c|c|}
\hline Num. of Elements & Throughput Decrease (\%) \\
\hline 4 & 4.08 \\
\hline 8 & 7.14 \\
\hline
\end{tabular}

possibility that one of the nodes that participates in a data transmission (as either transmitter or receiver) moves outside the directional range of the other. Thus, the packet under transmission is lost. On the other hand, there is a possibility that an entry in the Location Table for a specific node can be temporarily out of date. Thus, the node may activate or deactivate the D-NAV for the beam toward the specific node in a wrong way, destroying in this way an ongoing communication. Very soon, the node receiving new frames from the specific neighbor will update the wrong entry and will cancel the inefficiency. Both these facts lead to the degradation of the throughput. However, the modest levels of degradation demonstrate the viability of our protocol in mobile scenarios.

Table 5 depicts the percentage (in percent) of the throughput decrease in the cases of four and eight elements. As we can see, the throughput decrease is smaller in the case of four elements than in the case of eight elements. This is due to the fact that, in the case of eight elements, a node transmits with slighter beams and, thus, the possibility of missing the mobile receiver is higher. Similarly, there is a higher possibility for an entry in the Location Table to be temporarily out of date.

In our last experiment, we examine the reaction of our protocol in different levels of mobility. Again, we run the previous random scenario of 60 nodes. Now, every node has four elements. We run the simulation for two cases: In the first case, the nodes have low mobility, and in the second, they have high mobility. The throughput is depicted in Fig. 13.

As we can see in this figure, the degradation in the throughput increases as the mobility of the nodes increases. This is due to the same facts we described in the previous mobility scenario. However, the modest levels

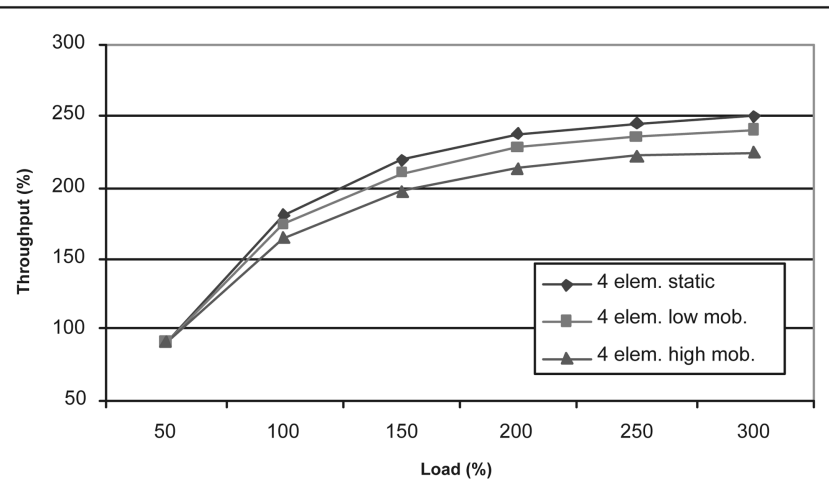

Fig. 13. The effect of mobility in the random scenario of 60 nodes. 
of degradation in this case also demonstrate the adaptability of our protocol in several levels of mobility.

\section{Conclusions}

In this work, we propose a MAC protocol suitable for networks with directional antennas. Our protocol, utilizing a new scheme for the broadcasting of RTS, employs only directional transmissions, increasing in this way the coverage area. Particularly, the proposed algorithm is based on a CDR that scans the area around the transmitter, informing the neighbors of the intended communication. Using a simple and effective scheme, the neighbors decide for their transmission differentiation in order not to destroy the ongoing transmission. In this way, there is a strong decrease in the hidden-terminal problem. The protocol does not assume any knowledge of the neighbors' locations. Due to the dynamic nature of the functionality of the protocol, it behaves efficiently in an environment with static, as well as mobile, users. The previous features result in an efficient integrated scheme that can be implemented easily.

\section{REFERENCES}

[1] A. Chandra, V. Gummalla, and J. Limb, "Wireless Medium Access Control Protocols," IEEE Comm. Surveys and Tutorials, vol. 3, no. 2, 2000.

[2] R. Choubhury and N. Vaidya, "Impact of Directional Antennas on Ad Hoc Routing," Proc. Eighth Int'l Conf. Personal Wireless Comm. (PWC '03), 2003.

[3] R. Choubhury and N. Vaidya, "Deafness: A MAC Problem in Ad Hoc Networks When Using Directional Antennas," Proc. 12th IEEE Int'l Conf. Network Protocols (ICNP '04), 2004.

[4] R.R. Choudhury, X. Yang, R. Ramanathan, and N.H. Vaidya, "Using Directional Antennas for Medium Access Control in Ad Hoc Networks," Proc. ACM MobiCom, 2002.

[5] M. Cooper and M. Goldburg, "Intelligent Antennas: Spatial Division Multiple Access," Ann. Rev. of Comm., pp. 999-1902, 1976.

[6] W.M. Moh, D. Yao, and K. Makki, "Wireless LAN: Study of Hidden Terminal Effect and Multimedia Support," Computer Comm. and Networks, 1998.

[7] T. ElBatt, T. Anterson, and B. Ryu, "Performance Evaluation of Multiple Access Protocols for Ad Hoc Networks Using Directional Antennas," Proc. IEEE Wireless Comm. and Networking Conf. (WCNC '03), 2003.

[8] G.J. Foschini and M.J. Gans, "On Limits of Wireless Communications in a Fading Environment When Using Multiple Antennas," Wireless Personal Comm., 1998.

[9] C.L. Fullmer and J.J. Garcia-Luna-Aceves, "Solutions to Hidden Terminal Problems in Wireless Networks," Proc. ACM Conf. Applications, Technologies, Architectures, and Protocols for Computer Commun. (SIGCOMM '97), 1997.

[10] M. Horneffer and D. Plassmann, "Directional Antennas in Mobile Broadband Systems," Proc. IEEE INFOCOM, 1996.

[11] IEEE Standard 802.11, Part 11: Wireless LAN Medium Access Control (MAC) and Physical Layer (PHY) Specifications, IEEE, 1999.

[12] P. Karn, "MACA-A New Channel Access Method for Packet Radio," Proc. Ninth ARRL Computer Networking Conf., 1990.

[13] Y.B. Ko, V. Shankarkumar, and N.H. Vaidya, "Medium Access Control Protocols Using Directional Antennas in Ad Hoc Networks," Proc. IEEE INFOCOM, 2000.

[14] T. Korakis, G. Jakllari, and L. Tassiulas, "A MAC Protocol for Full Exploitation of Directional Antennas in Ad-Hoc Wireless Networks," Proc. ACM MobiHoc, 2003.

[15] C. Liberti and T.S. Rappaport, Smart Antennas for Wireless Communication: IS-95 and Third Generation CDMA Applications, chapter 1. Prentice Hall, 1999.

[16] A. Nasipuri, S. Ye, J. You, and R.E. Hiromoto, "A MAC Protocol for Mobile Ad-Hoc Networks Using Directional Antennas," Proc. IEEE Wireless Comm. and Networking Conf. (WCNC '00), 2000.
[17] B. O'Hara and A. Petrick, 802.11 Handbook: A Designer's Companion, chapter on medium access control. IEEE Press, 1999.

[18] Radio/Wireless Models, OPNET, http://www.opnet.com/, 2007.

[19] T.S. Rappaport, Wireless Communication: Principles and Practice, chapter 1. Prentice Hall, 1996.

[20] R. Ramanathan, "On the Performance of Ad Hoc Networks with Beamforming Antennas," Proc. ACM MobiHoc, 2001.

[21] M. Takai, J. Martin, A. Ren, and R. Bagrodia, "Directional Virtual Carrier Sensing for Directional Antennas in Mobile Ad Hoc Networks," Proc. ACM MobiHoc, 2002.

[22] S. Yi, Y. Pei, and S. Kalyanaraman, "On the Capacity Improvement of Ad Hoc Wireless Networks Using Directional Antennas," Proc. ACM MobiHoc, 2003.

[23] T. Yum and K. Hung, "Design Algorithms for Multihop Packet Radio Networks with Multiple Directional Antennas," IEEE Trans. Comm., vol. 40, no. 11, 1992.

[24] J. Zander, "Slotted ALOHA Multihop Packet Radio Networks with Directional Antennas," Electronics Letters, vol. 26, no. 25, 1990.

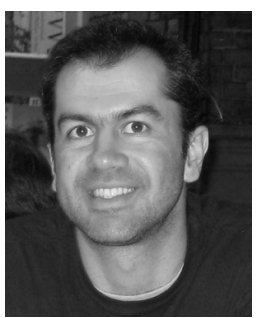

Thanasis Korakis received the BS and MS degrees in informatics and telecommunications from the University of Athens, Greece, in 1994 and 1997, respectively, and the $\mathrm{PhD}$ degree in computer and communication engineering from the University of Thessaly, Greece, in 2005 . He is a research assistant professor in the Electrical and Computer Engineering Department, Polytechnic University. He is also affiliated with the New York State Center for Advanced Technologies in Telecommunications (CATT) and the Wireless Internet Center for Advanced Technology (WICAT), Polytechnic University, where he was a research scientist from 2005 to 2006. In the summer of 2004, he was a visiting researcher in the Computer Science and Engineering Department, University of California, Riverside. His research focuses on access layer protocols, cooperative networks, directional antennas, quality of service provisioning, and network management. He is a member of the IEEE.

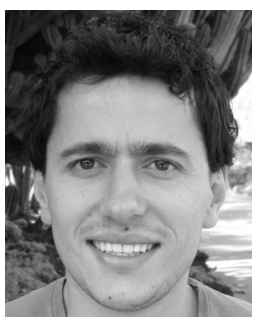

member of the IEEE.
Gentian Jakllari received the BS degree in computer science from the University of Ioannina, Greece, and the MSc degree in computer science from the University of California, Riverside. $\mathrm{He}$ is a $\mathrm{PhD}$ candidate in the Computer Science Department, University of California, Riverside. His research interests are in the field of wireless networking with an emphasis on the design, evaluation, and implementation of crosslayer protocols and algorithms. He is a student

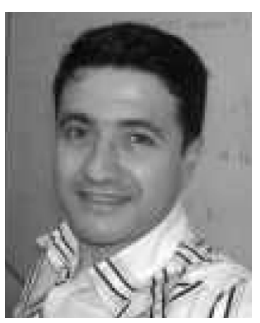

Leandros Tassiulas received the diploma in electrical engineering from the Aristotelian University of Thessaloniki, Greece, in 1987, and the $\mathrm{MS}$ and $\mathrm{PhD}$ degrees in electrical engineering from the University of Maryland, College Park, in 1989 and 1991, respectively. He has been a professor in the Department of Computer and Telecommunications Engineering, University of Thessaly, since 2002. He has held positions as an assistant professor at the Polytechnic University, New York, from 1991 to 1995, as an assistant and associate professor at the University of Maryland from 1995 to 2001, and as a professor at the University of Ioannina, Greece, from 1999 to 2001. His research interests are in computer and communication networks with an emphasis on fundamental mathematical models, architectures, and protocols of wireless systems, sensor networks, high-speed Internet, and satellite communications. He received a US National Science Foundation (NSF) Research Initiation Award in 1992, a US NSF Faculty Early Career Development (CAREER) Award in 1995, an Office of Naval Research Young Investigator Award in 1997, and a Bodosaki Foundation Award in 1999. He also received the INFOCOM 1994 Best Paper Award and the INFOCOM 2007 Achievement Award. He is a fellow of the IEEE. 